ORIGINAL ARTICLE

\title{
Audit of Oral Histopathology Service at a Nigerian Tertiary Institution over a 24-Year Period
}

\author{
Akindayo O. Akinyamoju ${ }^{1 *}$, Bukola F. Adeyemi ${ }^{1}$, Akinyele O. Adisa ${ }^{1}$, Chidinma \\ N. Okoli ${ }^{2}$
}

OPEN ACCESS

Citation: Akindayo O. Akinyamoju, Bukola F. Adeyemi, Akinyele O. Adisa, Chidinma N. Okoli. Audit of Oral Histopathology Service at a Nigerian Tertiary Institution over a 24-Year Period. J Health Sci 2017; 27(4):383 doi: http://dx.doi.org/10.4314/ejhs.v27i4.9 Received: February 13, 2017

Accepted: February 24, 2017

Published: July 1, 2017

Copyright: (@) 2017 Akinyamoju, A.O., et al. This is an open access article distributed under the terms of the Creative Commons Attribution License, which permits unrestricted use, distribution, and reproduction in any medium, provided the original author and source are credited.

Funding: Nil

Competing Interests: The authors declare that this manuscript was approved by all authors in its form and that no competing interest exists.

Affiliation and Correspondence: ${ }^{1}$ Department of Oral Pathology, Faculty of Dentistry, College of Medicine, University of Ibadan/ University College Hospital Ibadan, Nigeria

${ }^{2}$ Department of Oral Pathology,

University College Hospital, Ibadan, Nigeria

*Email: akindayo2002@yahoo.com

\section{ABSTRACT}

BACKGROUND: Biopsies are often essential for definitive diagnosis of oro-facial lesions and are a part of oral diagnostic procedures carried out in histopathology laboratories. At present, there is paucity of literature on the audit of oral histopathology services in Nigeria. The objectives of this study were to determine the prevalence of biopsied oral lesions in a Nigerian tertiary institution. Also to profile the usage of oral pathology service and to identify challenges that may be present in an oral histodiagnostic service.

METHODS: This was a retrospective study performed at the Oral Pathology Department of the University of Ibadan/University College Hospital, Ibadan, Nigeria. Reports of all biopsies submitted at the Oral Pathology laboratory, for the period 1990-2014, were reviewed and data extracted. Descriptive analysis was done using SPSS software, version 20.

RESULTS: The total number of reports was 1,998; invalid reports constituting 220(11\%) were subsequently excluded leaving

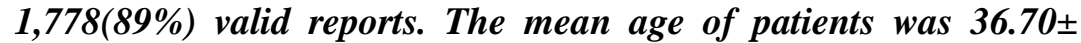
19.79, while the peak age of presentation was in the $3^{\text {rd }}$ decade. Male to female ratio was 1:1.1, and the mandible was the most common site of lesions 619(34.8\%). These services were mainly utilized by oral surgeons (83.9\%) and ameloblastoma (11.5\%) was the most frequently diagnosed lesion. CD45 (16.7\%) was the most frequently requested immuno-diagnostic test.

CONCLUSION: Biopsied oral lesions were more prevalent in females, while oral and maxillofacial surgeons utilized these services the most. Inadequate biopsy specimens or unrepresentative specimens and deficient documentation were challenges identified in this study.

KEYWORDS: Oral, Biopsy, Histopathology Service, Nigerian 


\section{INTRODUCTION}

Biopsies are often essential for definitive diagnosis of oro-facial lesions (1). This is due to the complex relationship of the various structures within the head and neck region, such as jaws, teeth and salivary gland amongst many others (2). However, certain principles guide the provision of a successful biopsy; most importantly the submission of a representative sample to the laboratory, as well as the preservation of excised tissue (3).

At present, oral histopathology services are a part of oral diagnostic procedures carried out in histopathology laboratories of tertiary institutions in Nigeria particularly at foremost dental schools located in the Southwest and Midwest regions. At the University of Ibadan/University College Hospital Ibadan (UCH), oral histopathology service was established in 1989. Prior to this time, these services were provided by general pathologists. Thus, the oral pathology laboratory of the University of Ibadan/University College Hospital, Ibadan, is an important oral pathology reference center in Oyo State, Southwest Nigeria. Specimens of oro-facial pathologies are received from general practitioners and various specialists within and outside the hospital as well as from neighbouring states.

These services are provided by oral pathologists who are lecturers/consultants, licensed by the Medical and Dental Council of Nigeria and are fellows of recognized postgraduate training colleges in Nigeria and West Africa. This is similar to what obtains worldwide where the practice of oral pathology is predominantly at tertiary/teaching institutions (4). Therefore, oral histopathology service provides a diagnostic laboratory for licensed practitioners and teaching materials for training both oral pathologists and dental students while serving as an archive of research material (3). However, oral pathology services may be scarce across Africa as previous reports have shown a shortage of pathology services in Sub-Saharan Africa $(4,5,6)$, with dependence on general pathologists to do the oral histopathological diagnosis (7).
Slight variation has been observed in the prevalence of oral lesions from one geographic region to the other, probably due to variations in culture, habits, environmental pollutants and genetic composition $(8,9,10)$. In a survey of oral and maxillofacial biopsies by Ali et al., malignant lesions accounted for $9.9 \%$ of lesions (11) while Oliveira e Silva et al., and Moridani et al., reported $6.32 \%$ and $2.38 \%$ respectively $(3,10)$. On the contrary, an East African study reported a high prevalence of malignancies $(67.28 \%)$, which was due to a selective inclusion of neoplastic lesions only (7).

Furthermore, researchers have studied the profile usage of oral histopathology services. Previous studies have reported a predominance of specialists over general practitioners in the utilization of these services $(3,12)$. This was attributed to the higher number of specialists at tertiary centers where oral pathology services are available. In addition, referral of patients who need biopsies by general practice dentists to specialists contributes to the higher usage by specialists (3).

Oral histopathology services in a developing country may face various challenges that could undermine its effective functioning. Jargin observed that histological specimen were often not clearly marked and lacked clinical information, requiring the histopathologist to request these information from patients or their relatives (13). As well, histodiagnosis of poorly differentiated neoplasms could be quite challenging, especially when diagnosis is made based on microscopic, clinical and radiographic features alone $(13,14)$. In recent times, ancillary studies like immunohistochemistry have greatly assisted in the characterization of poorly differentiated and undifferentiated neoplasms that were a diagnostic challenge $(15,16)$.

At present, there is paucity of literature on the audit of oral histopathology services in Nigeria. A review of these services would contribute to knowledge, provide information on the burden of oral diseases in this region while providing data for effective policy formulation and suggest measures to improve service delivery. Therefore, the objectives of this study were to determine the

DOI: http://dx.doi.org/10.4314/ejhs.v27i4.9 
prevalence of biopsied oral lesions, to determine the profile usage of the oral pathology laboratory service at the UCH Ibadan and to identify the challenges that may be present in an oral histodiagnostic service.

\section{MATERIALS AND METHODS}

This retrospective study was performed at the Oral Pathology Service of the University of Ibadan/University College Hospital, Ibadan. Reports of all biopsies submitted at the Oral Pathology laboratory, for the period 1990-2014 were reviewed. Data were extracted from histopathology reports using a data collection form which included age, gender, site of lesion and source of request. Histopathological diagnosis was recorded as reported in the biopsy report and was standardized using current terminologies. Repeat and recurrent biopsies were recorded once if the diagnoses remained the same, while the later diagnosis was documented if different.

Lesions were grouped into either benign, premalignant or malignant and further categorized into 16 broad diagnostic groups as follows: reactive lesions, cystic lesions, pulp and periapical lesions, giant cell lesions, fibro-osseous lesions, odontogenic tumours, epithelial tumours, salivary gland diseases, soft tissue tumours; salivary gland tumours; heamato-lymphoid neoplasms, inflammatory/microbial diseases; ulcerative lesions, collision tumours, normal tissue and miscellaneous. Reports that had inadequate information with regard to demographic data, clinical information or had indeterminable or imprecise diagnosis (unrepresentative biopsy specimen) were identified and analyzed before exclusion from further analysis. These reports were defined as those with one or more of the following missing information: age, gender, site of lesion, clinical description of lesion and clinical diagnosis. Likewise, reports that were unsigned as well as those that were inconclusive or without a final submission were recorded to be inadequate. Also, challenging cases (poorly differentiated and undifferentiated neoplasms) that required immunohistochemistry for diagnosis were also extracted from the records and included in the study. Descriptive analysis was done using SPSS software, version 20. Ethical approval was obtained from the UI/UCH Ethical Review Board (UI/EC/16/0284).

\section{RESULTS}

Over the study period, 1,998 histodiagnostic reports were available, of which $220(11 \%)$ either had deficient information in the demographics and/or clinical information, or were imprecise in diagnosis constituting $4.2 \%$ and $6.8 \%$ respectively of the entire sample. These insufficient reports were mainly from samples sent for histodiagnosis by oral and maxillofacial surgeons (78.6\%) and general practitioners $(10 \%)$. Thus, they could no longer be used in the study. These were subsequently excluded from further analysis (Figure 1).

Correctly filled request forms and written reports were $1,778(89 \%)$ with a mean of 74 cases diagnosed each year. The mean age was $36.70 \pm$ 19.79, while peak age group was the third decade $(20.5 \%)$. Females $(51.5 \%)$ constituted the most affected gender and male to female ratio was 1:1.1. Thirty-six different sites were recorded as biopsy sites, and most commonly biopsied sites were the mandibular bone $(34.8 \%)$, maxilla $(21.2 \%)$, gingivae $(12.3 \%)$, palate $(7.8 \%)$ and tongue $(4.4 \%)$, while other sites constituted $19.5 \%$. About $74.4 \%$ of the biopsies were reported as benign lesions; $25.5 \%$ were malignant while pre-malignant lesions constituted $0.1 \%$. Most of the requests were from oral and maxillofacial surgeons (83.9\%), followed by oral pathologists/oral medicine specialists $(8 \%)$, paediatric dentists $(2.4 \%)$, conservative dentists $(2.1 \%)$ and general practice dentists $(2.1 \%)$ (Table $1)$.

DOI: http://dx.doi.org/10.4314/ejhs.v27i4.9 


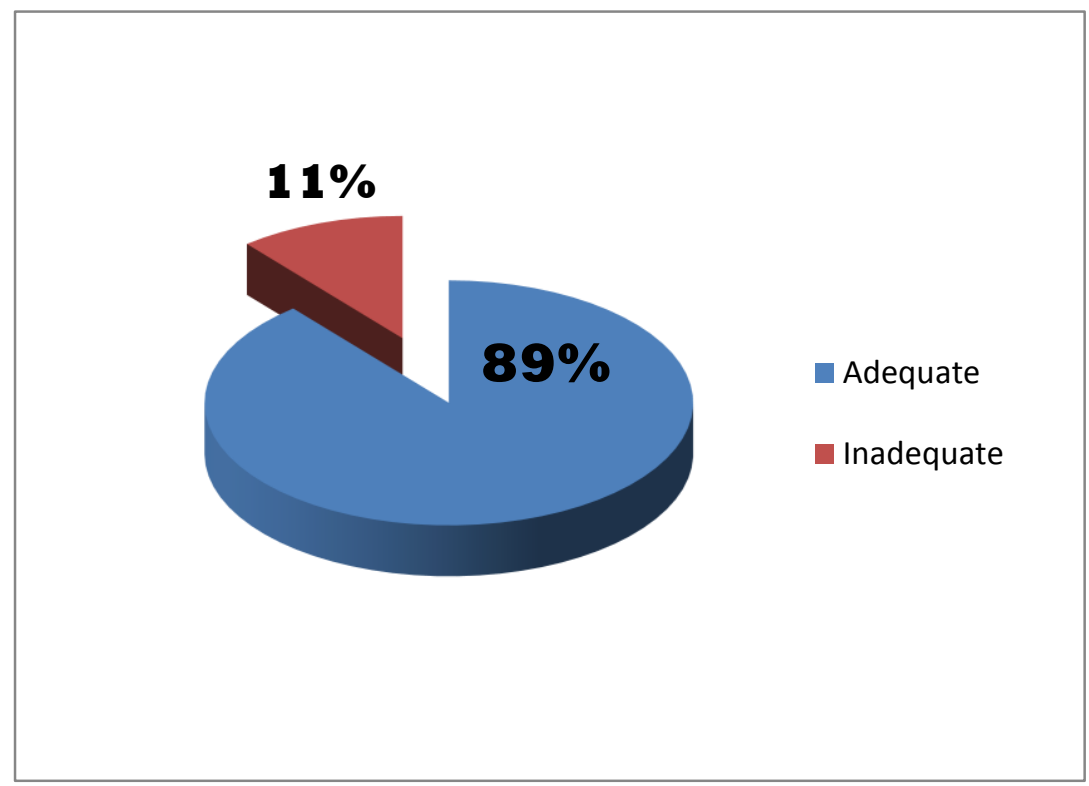

Figure 1: Adequacy of histopathology records

Table 1: Source of request

\begin{tabular}{lcc}
\hline Source of Request & Number of Request & Percent \\
\hline Oral \& Maxillofacial Surgery & 1491 & $83.9 \%$ \\
Oral Pathology/Oral Medicine & 142 & $8 \%$ \\
Paediatric Dentistry & 42 & $2.4 \%$ \\
General Practice Dentists & 38 & $2.1 \%$ \\
Conservative Dentistry & 38 & $2.1 \%$ \\
Periodontology & 20 & $1.12 \%$ \\
*Other Specialist Within & 6 & $0.33 \%$ \\
**Other Specialist Outside & 1 & $0.05 \%$ \\
Total & $\mathbf{1 7 7 8}$ & $\mathbf{1 0 0 \%}$ \\
\hline
\end{tabular}

*ENT; Plastic Surgery; Orthodontics; General Pathology and Prosthodontics

**Federal Medical Centre

Overall, 207 different diagnoses were made from the lesions sent for histopathology. The most frequently diagnosed category of lesions were reactive lesions $(23.1 \%)$, odontogenic tumours
(18.1\%) and epithelial tumours (12.4\%) (Table 2), while the most frequently diagnosed lesions were ameloblastoma (11.5\%), squamous cell carcinoma $(10.4 \%)$ and pyogenic granuloma $(8.5 \%)$ (Table $3)$.

DOI: http://dx.doi.org/10.4314/ejhs.v27i4.9 
Table 2: Categorization of diagnosis

\begin{tabular}{lcc}
\hline Category of Lesion & Frequency & Percent \\
\hline Reactive Lesions (RL) & 411 & $23.1 \%$ \\
OdontogenicTumours (OT) & 321 & $18.1 \%$ \\
Epithelial Tumours (ET) & 220 & $12.4 \%$ \\
Salivary Gland Tumours (SGT) & 163 & $9.2 \%$ \\
Soft Tissue Tumours (STT) & 159 & $8.9 \%$ \\
Fibroosseous Lesions (FOL) & 149 & $8.4 \%$ \\
Pulp and Periapical Lesions (PPL) & 120 & $6.7 \%$ \\
Cystic Lesions (CL) & 70 & $3.9 \%$ \\
Heamatolymphoid Neoplasms (HLN) & 56 & $3.1 \%$ \\
Salivary Gland Diseases (SGD) & 33 & $1.9 \%$ \\
Inflammatory/ Microbial Diseases (IMD) & 24 & $1.3 \%$ \\
Giant Cell Lesions (GCL) & 21 & $1.2 \%$ \\
Normal Tissue (NT) & 17 & $1.0 \%$ \\
Ulcerative Lesions (UL) & 5 & $0.3 \%$ \\
Collision Tumours (CT) & 4 & $0.2 \%$ \\
*Miscellaneous & 5 & $0.3 \%$ \\
\hline Total & $\mathbf{1 7 7 8}$ & $\mathbf{1 0 0 \%}$ \\
\hline
\end{tabular}

*Miscellaneous -Harmatoma (1), Eosinophilic Granuloma (1), Amyloidosis (1), Hyperplastic oral epithelium (2)

All in all, challenging cases that required the use of immunohistochemistry for confirmation were $41(2.3 \%)$. They consisted of sarcomas $(46.4 \%)$, suspected lymphomas (26.8\%), poorly differentiated carcinoma (24.4\%) and vascular lesions $(2.4 \%)$. Thirteen different antibodies were requested for the immunohistochemical tests including vimentin, leucocyte common antigen (LCA), CD3, CD5, CD20, CD23, CD34, neuron specific enolase (NSE), cytokeratins (AE1/AE3), S100, myogenin, desmin and HMB 45. Figure 2 shows the frequency distribution of different antibodies requested for immunohistochemical tests. 


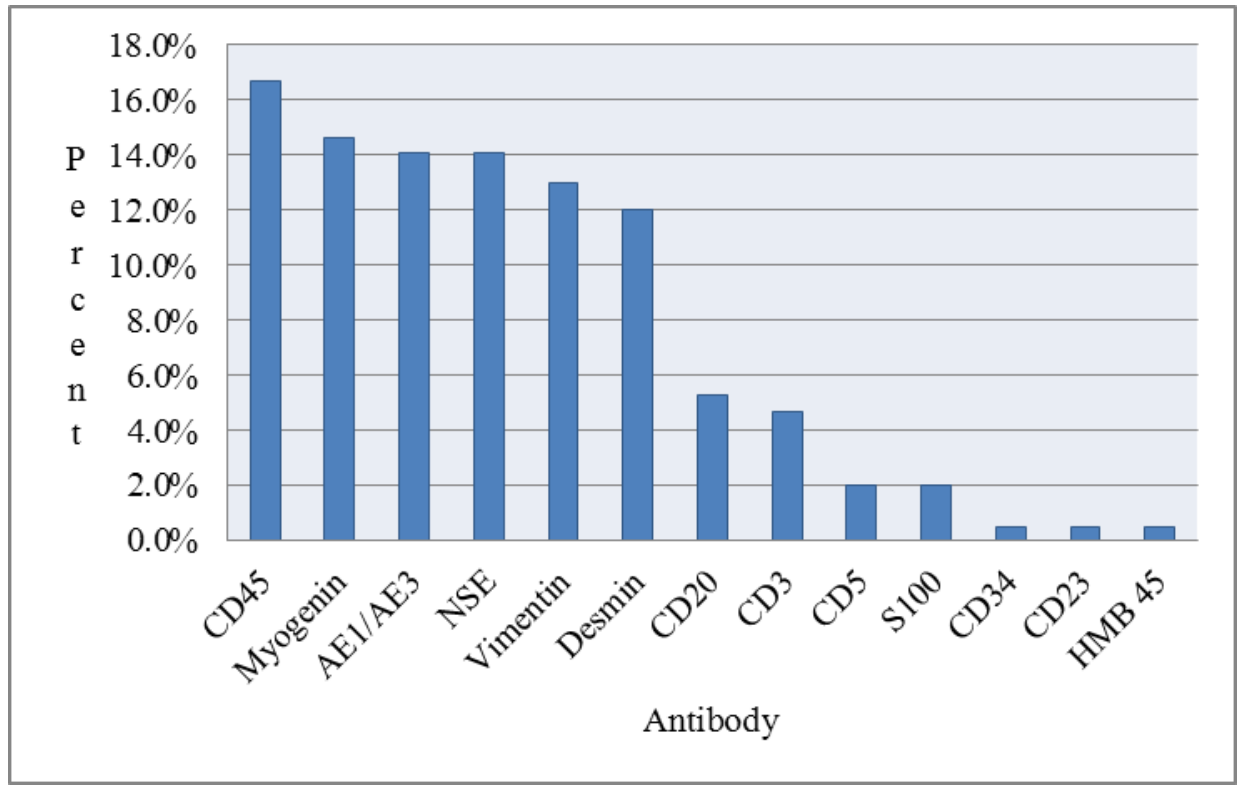

Figure 2: Antibodies requested for immunohistochemical tests

Table 3: Frequency of 20 commonly diagnosed lesions

\begin{tabular}{lcc}
\hline Diagnosis & Frequency & Percent \\
\hline Ameloblastoma & 204 & $11.5 \%$ \\
Squamous Cell Carcinoma & 185 & $10.4 \%$ \\
Pyogenic Granuloma & 151 & $8.5 \%$ \\
Ossifying Fibroma & 70 & $3.9 \%$ \\
Chronic Inflammation & 64 & $3.6 \%$ \\
Apical Cyst & 61 & $3.4 \%$ \\
Apical Granuloma & 56 & $3.1 \%$ \\
Fibromyxoma & 51 & $2.9 \%$ \\
Fibroma & 50 & $2.8 \%$ \\
Fibrous Dysplasia & 50 & $2.8 \%$ \\
Fibrous Epulis & 46 & $2.6 \%$ \\
Pleomorphic Adenoma & 40 & $2.2 \%$ \\
Adenoid Cystic Carcinoma & 40 & $2.2 \%$ \\
Granulation tissue & 37 & $2.1 \%$ \\
Osteogenic Sarcoma & 30 & $1.7 \%$ \\
Burkitt's Lymphoma & 29 & $1.6 \%$ \\
Muco-Epidermoid Carcinoma & 26 & $1.5 \%$ \\
Keratocystic OdontogenicTumour & 19 & $1.1 \%$ \\
Non- Hodgkins Lymphoma & 17 & $1.0 \%$ \\
Osteoma & 17 & $1.0 \%$ \\
*Others & 535 & $30.1 \%$ \\
Total & $\mathbf{1 7 7 8}$ & $\mathbf{1 0 0 \%}$ \\
\hline
\end{tabular}

*Others consist of 187 different diagnoses that each recorded less than $1 \%$ prevalence

DOI: http://dx.doi.org/10.4314/ejhs.v27i4.9 


\section{DISCUSSION}

There are reports in literature which examined the prevalence of biopsied oral lesions $(3,11)$ while others determined the prevalence of oral biopsies amongst different groups and anatomical sites $(17,18)$. In this study, the number of biopsies sent for histodiagnosis over the study period was relatively less compared to what was recorded in other studies $(3,11)$. This study recorded 1,998 requests over a 24 year study period while Oliveira e Silva et al., and Ali et al., reported 13, 522 and 3,150 requests respectively over a 10 year study period $(3,11)$. The higher numbers reported by these studies may be due to larger catchment areas in these studies compared to that of this study and may also be due to some oral samples being sent to general pathologists within the early phase of the study period. In addition, not all excised tissue from the oral cavity especially attached periapical tissue are sent for microscopic examination by dental practitioners (19) and this may be more prevalent in a developing country.

The mean age of $36.70 \pm 19.79$ recorded in this study is similar to that obtained from previous studies. Ali et al. (11), and Moridani et al. (10), reported mean ages of $34.90 \pm 16.72$ and 38 years respectively. However, the peak age range of third to fifth decade reported by Moridani et al. (10) and fifth decade reported by Takashima and Etges (20) differ from the third decade recorded in this study. Also, this study recorded a female preponderance of biopsied oral lesions of $51.5 \%$, similar to that of Ali et al. (51.4\%) (11), Takashima and Etges (59\%) (20) and Moridani et al. (53.3\%) (10). In contrast to this, Chidzonga et al., reported a higher male predilection of $54.5 \%$ in their study (21). These subtle differences in the demographics of biopsies submitted for histopathology in these studies may be due to differences in how patients in different climes perceive and access oral health. In this study, the most common biopsy location was the mandible $(34.8 \%)$. This is similar to the study by Moridani et al., who also reported the mandible as the most common biopsy site (10). This is contrary to a previous study that recorded maxillary and mandibular gingivae as the most common sites (20). Also, this study recorded a predominantly benign nature of biopsied lesions (74.4\%), similar to the study by Oliveira e Silva et al., who reported a higher fraction $(93.2 \%)$ of benign lesions (3). However, this study recorded a higher fraction of malignancies $(25.5 \%)$ compared to studies by Ali et al. (9.9\%) (11), Oliveira e Silva et al. (6.3\%) (3) and Moridani et al. (2.4\%) (10). This may be attributed to variation in the cases utilized in these studies. In addition, the present study was conducted at a referral centre with facilities for cancer management. Thus malignant lesions are more likely to be referred to this centre than benign lesions (22).

Furthermore in this study, lesions categorized as reactive lesions were the most frequent group of lesions diagnosed, constituting $23.1 \%$ of the entire diagnosis. A similar finding was seen in a previous study by Moridani et al., who recorded $21.5 \%$ in their study (10). The high proportion of reactive lesions in these studies may not be unconnected with the high susceptibility of the oral mucosa to chronic irritation. The most frequently diagnosed lesion in this study was ameloblastoma constituting $11.5 \%$ of the entire biopsies. This finding is at variance with previous studies from other climes which reported fibrous epulis $10.3 \%$ (23), fibrous hyperplasia $20.9 \%$ (8) and inflammatory fibrous hyperplasia $17 \%$ (20) as frequently diagnosed lesions. The reason for this disparity may be due to high incidence of ameloblastoma in Africans $(24,25)$, and also the cases in some previous studies were skewed to mucosa and submucosa pathology $(20,23)$.

On requests for histopathology of excised tissue, about $97.9 \%$ requests for histodiagnosis were from specialists, $83.9 \%$ of which were oral and maxillofacial surgeons. This trend is similar to observations by Wan and Savage in a study of the usage pattern of biopsy and histopathology services, where they reported that $89.1 \%$ of biopsies were requested by specialists (12). However, they reported a predominance of oral medicine specialists $(39.9 \%)$ over oral and maxillofacial surgeons (29\%), which is at variance with our study. This difference may be attributable to variation in the study location of both studies. This study was conducted in a tertiary institution where oral and maxillofacial surgeons are more

DOI: http://dx.doi.org/10.4314/ejhs.v27i4.9 
likely to perform biopsy procedures compared to other specialists, while Wan and Savage conducted their study in two oral medicine clinics. However, both studies recorded a lower proportion of biopsy service usage by general dental practitioners, $2.1 \%$ in this study and $10.9 \%$ by Wan and Savage (12) respectively. This is because general dental practitioners often refer challenging cases for specialist attention and only biopsy selected cases. In addition, they may not encounter oral lesions requiring biopsies frequently, since patients are more likely to present at tertiary centers with such lesions (12).

With regard to challenges that may confront an oral pathology service as seen in this study, about $11.01 \%$ of the entire histopathological reports were excluded from further analysis either due to incomplete records or imprecise diagnosis. While in studies by Franklin and Jones and Oliveira e Silva et al., $0.26 \%$ and $0.5 \%$ of cases respectively had insufficient information or histologic features to make a definitive diagnosis $(3,8)$. Also, clinical information plays an important role in making a definitive diagnosis but was omitted in the laboratory request form of some cases in this study. For example, pyogenic granuloma and pregnancy epulis have essentially the same histologic appearance, and without clinical information, accurate diagnosis may be difficult (26). In 2011, Jargin in a letter to the editor of the Turkish Journal of Pathology identified lack of clinical information as a challenge in histodiagnostic services in a developing country (13). Similarly, obstacles to collecting accurate data in sub-Saharan Africa have been elucidated in a previous study (27). These shortcomings, either deliberate or due to negligence and when in sizable proportions, are capable of rendering data immaterial, thus undermining the use of such data.

Furthermore, challenges were encountered in the histodiagnosis of certain lesions in the oro-facial complex, given the overlap of histological features that exist amongst some groups. This necessitated the use of immunohistochemical staining as an ancillary test in $2.3 \%$ of cases. This was similar to $2.1 \%$ cases requiring immunochemical diagnosis in a study by Ajura et al. (15), but in contrast with findings by Oliveira e Silva et al., where more cases $(3.4 \%)$ were sent for immunohistochemical staining (3). Also, this study revealed that the requested antibodies and their indications were similar to those seen in previous studies $(3,15)$. The lower number of cases for ancillary investigations seen in the present study may be due to a relative lower number of routine histopathology cases seen. Another reason is that facilities for immunohistochemical analysis in this centre existed at the latter half of the study period. Also, immunohistochemical stains are still limited and seldom used in most histopathology laboratories in Africa, due to various reasons highlighted in reports by Adeyi (4) and Patel et al., (28), with pathologist often relying wholly on histodiagnosis $(4,28)$. Adeyi and Patel et al., in their studies suggested models for the improvement of histodiagnostic services in Africa including mentoring and collaboration with institutions in the developed world $(4,28)$. These measures, when implemented, would help improve service delivery and patient care.

The present study reported an analysis of cases submitted to a surgical oral pathology laboratory as well as described the prevalence of biopsied oral lesions submitted for histodiagnoses rather than the prevalence of these lesions in the region. The oral biopsy cases in our archives may not constitute the entirety of oral biopsy lesions seen over the study period, but most likely comprise the majority because the study location is the only oral pathology reference center for Oyo State, Southwest, Nigeria. Similarly, cases that did not meet the inclusion criteria may be substantial enough to influence some of the results obtained in this study.

In conclusion, this study showed that biopsied oral lesions were more prevalent in females and in patients in the third decade of life. The mandible was the most biopsied site and the majority of the biopsies were benign lesions. Also, profile of usage showed that oral and maxillofacial surgeons made the highest number of requests for histopathology review. This study likewise revealed some challenges that may be encountered in an oral pathology service, which include inadequate biopsy specimens or unrepresentative

DOI: http://dx.doi.org/10.4314/ejhs.v27i4.9 
specimens and deficient documentation that render records unfit for research and definitive diagnosis. Continuous education for all dental practitioners with regard to the need for all excised tissues to be sent for histopathological evaluation should be reemphasized. Likewise, better biopsy techniques, proper handling of tissues and provision of appropriate information for each case should be re-emphasized. These measures would ensure that more samples are submitted for histopathology as well as reduce the incidence of indeterminate diagnosis to the barest minimum.

\section{REFERENCES}

1. Regezi JA. Odontogenic cysts, odontogenic tumors, fibroosseous, and giant cell lesions of the jaws. Mod Pathol 2002; 15:3: 331-341.

2. Oliver RJ, Sloan P, Pemberton MN. Oral biopsies: methods and applications. Br Dent $\mathrm{J}$ 2004; 196:6:329-333.

3. Oliveira e SilvaKR, SiqueiraALL, CaldeiraPC, Guimarães de AbreuMHN, Ferreira de Aguiar MC.Profile of usage of a reference diagnostic service on oral pathology: a 10-year evaluation. BMC Health Serv Res 2014; 14:653-657.

4. Adeyi OA. Pathology services in developing countries-the West African experience. Arch Pathol Lab Med 2011;135(2):183-186.

5. Rambau PF. Pathology practice in a resourcepoor setting: Mwanza, Tanzania. Arch Pathol Lab Med. 2011;135(2):191-193.

6. Benediktsson H, Whitelaw J, Roy I. Pathology services in developing countries: A challenge. Arch Pathol Lab Med. 2007;131(11):16361639.

7. Kamulegeya A, Kalyanyama BM. Oral maxillofacial neoplasms in an East African population a 10 year retrospective study of 1863 cases using histopathological reports. BMC Oral Health 2008;8:19 doi: 10.1186/1472-6831-8-19.

8. Franklin CD, Jones AV. A survey of oral and maxillofacial pathology specimens submitted by general dental practitioners over a 30 -year period. Br Dent J 2006; 200:447-450.

9. Jones AV, Franklin CD. An analysis of oral and maxillofacial pathology found in adults over a 30-year period. J Oral Pathol Med 2006; 35:7:392-401.

10. Moridani SG, Shaahsavari F, Adeli MB. A 7year retrospective study of biopsied oral lesions in 460 Iranian patients. RSBO 2014; 11: 2:118-124.

11. Ali AA, Suresha CS, Al-Tamimi D, Al-Nazrc M, Atassi RA, Al-Rayese I, et al. A survey of oral and maxillofacial biopsies in the Eastern Province of Saudi Arabia: A 10 years' retrospective study. J Oral Maxillofac Surg Med Pathol 2013; 25:393-398.

12. Wan W, Savage NW. Biopsy and diagnostic histopathology in dental practice in Brisbane: usage patterns and perception of usefulness. Aust Dent J 2010; 55:162-169.

13. Jargin S. Histopathology in Iraq: Reliable Diagnostics in Spite of Shortages. (Letter to Editor).Türk Patoloji Dergisi 2011; 27: 2:177179.

14. Taylor CR. An exaltation of experts: concerted efforts in the standardization of immunohistochemistry. Appl Immunohistochem 1993; 1:232-243.

15. Ajura AJ, Sumairi I, Lau SH. The use of immunohistochemistry in an oral pathology laboratory. Malaysian J Pathol 2007; 29:2:101-105.

16. Adisa AO, Oluwasola AO, Adeyemi BF, Kolude B, Akang EEU, Lawoyin JO. Immunohistochemical analysis of undifferentiated and poorly-differentiated head and neck malignancies at a tertiary hospital in Nigeria. Head Neck Oncol 2010 2:33doi: 10.1186/1758-3284-2-33

17. Sengüven B, Bariş E, Yildirim B, Shuibat A, Özer Yücel O, Museyibov F, et al. Oral mucosal lesions: a retrospective review of one institution's 13-year experience. Turk J Med Sci 2015; 45:241-245.

18. Vale EBd, Ramos-Perez FMdM, Rodrigues GLC, Carvalho EJdA, Castro JFLd, Perez DEdC.et al. A review of oral biopsies in children and adolescents: a clinicopathological study of a case series. J Clin Exp Dent 2013; 5:3:e144-149. 
19. Mota-Ramírez A, Silvestre FJ, Simó JM. Oral biopsy in dental practice. Med Oral Patol Oral Cir Bucal 2007; 1: 12 (7):E504-510.

20. Takashima MR, Etges A. Epidemiological survey of biopsy performed in a residency program in bucco maxillofacial surgery.Rev Gaúcha Odontol 2012; 60: 3: 337-342.

21. Chidzonga MM, Lopez VM, Portilla Alvarez AL. Orofacial biopsies: a survey of 1723 cases seen over a 10 year period. Cent Afr J Med 1996; 42: 102-108.

22. Adeyemi, BF, Adekunle, LV, Kolude, BM, Akang, EEU, Lawoyin JO. Head and Neck Cancer- A Clinicopathological Study in a Tertiary Care Center.J Natl Med Assoc 2008; 100: 6: 690-697.

23. Tay ABG. A 5-year survey of oral biopsies in an oral surgical unit in Singapore: 1993-1997. Ann Acad Med Singapore 1999; 28:5:665-671.

24. Barnes L, Everson JW, Reichart P, Sidransky $\mathrm{D}$, editors. Pathology and genetics of head and neck tumours. IARC Press, Lyon 2005.
25. Mosadomi A. Odontogenic tumours in an African population. Analysis of twenty-nine cases seen over a 5-year period. Oral Surg Oral Med Oral Pathol 1975; 40(4):502-521.

26. Poh CF, Ng S, Berean KW, Williams PM, Rosin MP, Zhang L Biopsy and histopathologic diagnosis of oral premalignant and malignant lesions.J Can Dent Assoc2008; 74 (3):283-288.

27. Pullum TW. An assessment of age and date reporting in the DHS surveys, 1985-2003. DHS Methodological Reports. Calverton, MD: United States Agency for International Development. Report No.:5, 2006.

28. Patel K, Strother RM, Ndiangui F, et al. Development of immunohistochemistry services for cancer care in western Kenya: Implications for low- and middle-income countries. Afr J Lab Med. 2016;5:1:7 pages. doi: 10.4102/ajlm.v5i1.187 